\title{
QUALITY CONTROL FOR MARKOV CHAINS AND FREE BOUNDARY PROBLEMS ( ${ }^{\mathbf{1}}$ ) \\ BY
}

\author{
ROBERT F. ANDERSON AND AVNER FRIEDMAN
}

\begin{abstract}
A machine can manufacture any one of $n$ Markov chains $\boldsymbol{P}_{\boldsymbol{x}}^{\lambda}$ $(1<j<n)$; the $P_{x}^{\lambda}$ are defined on the space of all sequences $x=\{x(m)\}$ $(1<m<\infty)$ and are absolutely continuous (in finite times) with respect to one another. It is assumed that chains $P_{x}^{\lambda}$ evolve in a random way, dictated by a Markov chain $\theta(m)$ with $n$ states, so that when $\theta(m)=j$ the machine is producing $P_{x}^{\lambda}$. One observes the $\sigma$-fields of $x(m)$ in order to determine when to inspect $\theta(m)$. With each product $P_{x}^{\lambda}$ there is associated a cost $c_{j}$. One inspects $\theta$ at a sequence of times (each inspection entails a certain cost) and stops production when the state $\theta=n$ is reached. The problem is to find an optimal sequence of inspections. This problem is reduced, in this paper, to solving a certain free boundary problem. In case $n=2$ the latter problem is solved.
\end{abstract}

0 . Introduction. Let $X$ be a fixed countable subset of the real line. Let $\theta(t)$ $(t=0,1,2, \ldots)$ be a Markov chain with $n$ states $1,2, \ldots, n$, and with transition probability matrix $p_{i, j}$. With each state $i$ we associate a Markov chain $P_{x}^{\lambda_{1}}$ defined on the space $\Omega_{1}$ of sequences $\left(x_{0}, x_{1}, x_{2}, \ldots\right)$ where each $x_{i}$ varies in $X$. We assume that the $P_{x}^{\lambda_{i}}$ are distinct from each other and absolutely continuous (in finite time) with respect to one another. Denote by $E^{i, x}$ the expectation corresponding to the random evolution of the $P_{x}^{\lambda_{j}}$ in accordance with the chain $\theta(t)$ starting at $\theta=i$ and $x$.

Let $K_{1}, \ldots, K_{n-1}$ be given positive numbers. Let $c_{1}, \ldots, c_{n}$ be given nonnegative numbers and define a function $f(\theta)$ by $f(i)=c_{i}$ if $i=1$, $2, \ldots, n$. Let $\tau=\left(\tau_{1}, \tau_{2}, \ldots\right)$ be an increasing sequence of "inspection times" in the sense that $\tau_{i}$ assumes only nonnegative integer values and each set $\left(\tau_{i} \leqslant s\right)$ ( $s$ nonnegative integer) depends only on the coordinates $x_{0}, x_{1}, \ldots, x_{s}$ and on the knowledge of $\theta\left(\tau_{j}\right)$ for all $1 \leqslant j \leqslant i-1$.

Throughout this paper we shall use the notation

Received by the editors June 13, 1976.

AMS (MOS) subject classifications (1970). Primary 62N10, 90B25; Secondary 60J05, 60J75, 60K30, 90A15, 93E15, 93E99.

Key words and phrases. Markov chain, quality control, stopping time, inspection time, quasi variational inequality.

( $\left.{ }^{1}\right)$ This paper is partially supported by National Science Foundation Grant MPS 72-04959 A02.

(C) American Mathematical Society 1979 


$$
\int_{a}^{b} g(s) d s=g(a)+g(a+1)+\cdots+g(b)
$$

where $a, b$ are integers and $0 \leqslant a<b$.

Consider the cost function

$$
\begin{aligned}
J_{x}^{i}(\tau)= & E^{i, x}\left[K_{i}+\sum_{j=1}^{n-1} K_{j}\left[\sum_{l=1}^{\infty} I_{\theta\left(\tau_{l}\right)=j}\right]\right] \\
& +E^{i, x}\left[\int_{0}^{\tau_{1}-1} f(\theta(s)) d s+\sum_{j=1}^{n-1} \sum_{l=1}^{\infty} I_{\theta\left(\tau_{l}\right)=j} \int_{\tau_{l}-1}^{\tau_{l+1}-1} f(\theta(s)) d s\right] .
\end{aligned}
$$

The problem considered in this paper is to find and characterize a sequence of inspection times $\bar{\tau}=\left(\bar{\tau}_{1}, \bar{\tau}_{2}, \ldots\right)$ such that

$$
J_{x}^{i}(\bar{\tau})=\inf _{\tau} J_{x}^{i}(\tau)
$$

This is called a quality control problem. The same problem in the case of continuous-parameter Markov processes was studied by the authors in [1], [2]. The problem was reduced to solving a certain elliptic quasi variational inequality (q.v.i.). We shall establish a similar reduction also in the present setting of Markov chains. Analogously to the q.v.i. of [1], [2] we shall obtain here a "discrete" q.v.i. In the special case where $n=2$ we shall solve the q.v.i.

The development of this paper proceeds parallel to [2]. Some of the results follow similarly to [2], and these will be mentioned only briefly. There are, however, some novel features in the present Markov chain setting.

In $\$ 1$ we introduce the random evolution process $(x, \theta)$. We choose a model as in [2, Appendix] which displays very clearly the structure of this evolution.

In $\$ 2$ we introduce the $p$-process and prove results analogous to Theorems $2.1,2.2$ of [2]. The quality control problem is introduced in $\$ 3$, where it is reduced to solving a certain "discrete" q.v.i.

In $\$ 4$ we solve the q.v.i. in case $n=2$ under some monotonicity assumption.

1. The $(x, \theta)$ process. It will be convenient to denote the discrete parameter of various Markov chains by $t$; thus the parameter $t$ will take values $t=0,1$, $2, \ldots$ We fix a countable set $X$ of points on the real line and denote by $\Omega_{1}$ the space of all sequences $\omega=\left(x_{0}, x_{1}, x_{2}, \ldots\right)$ with $x_{i} \in X$. Viewing $\omega$ as a function $x=x(t)=x(t, \omega)$ on the nonnegative integers with values in $X$, we write $x_{t}=x(t)=x(t, \omega), t=0,1,2, \ldots$

Let $\theta(t)$ be a Markov chain with $n$ states $1,2, \ldots, n$ defined on a probability space $\Omega_{0}$ of all sequences $\omega^{\prime}=\left(\theta_{0}, \theta_{1}, \theta_{2}, \ldots\right)$ where each $\theta_{i}$ may take values $1,2, \ldots, n$. Viewing $\omega^{\prime}$ as a function $\theta=\theta(t)=\theta\left(t, \omega^{\prime}\right)$, we write $\theta_{t}=\theta(t)=\theta\left(t, \omega^{\prime}\right), t=0,1,2, \ldots$ Denote the transition probability matrix of $\theta(t)$ by $p_{i, j}$. 
Let $P_{x}^{\lambda_{i}}(i=1, \ldots, n)$ be $n$ distinct Markov chains defined on $\Omega_{1}$ and absolutely continuous (in finite time) with respect to one another. Denoting the transition probability matrix of $P_{x}^{\lambda}$ by $p_{j, k}^{\lambda}$ we then have, for each pair $(j, k)$,

$$
\text { either } p_{j, k}^{\lambda}=0 \text { for all } 1 \leqslant i \leqslant n \text { or } p_{j, k}^{\lambda}>0 \text { for all } 1 \leqslant i<n \text {. }
$$

We are interested in an explicit construction of the random evolution of the $P_{x}^{\lambda_{1}}$ in accordance with the law of $\theta(t)$. First we write down what, intuitively speaking, the transition probabilities should be:

$$
\begin{aligned}
& P_{i, x}(\theta(t)=j, x(t) \in B)=\sum_{\rho=0}^{t-1} \sum_{\left(i, \gamma_{1}, \ldots, \gamma_{\rho} j\right)} \\
& \cdot \sum_{u_{i}+u_{\gamma_{1}}+\cdots+u_{\gamma_{\rho}}<t} p_{i, i}^{u_{i}-1} p_{i, \gamma_{1}} p_{\gamma_{1}, \gamma_{1}}^{u_{\gamma_{1}}-1} p_{\gamma_{1}, \gamma_{2}} p_{\gamma_{2}, \gamma_{2}}^{u_{\gamma_{2}}-1} \cdots p_{\gamma_{\rho}, j} p_{j, j}^{t-u_{i}-u_{\gamma_{1}}-\cdots-u_{\gamma_{\rho}}} \\
& \cdot P_{x}^{\lambda_{i}} \otimes_{u_{i}}^{0} P_{x}^{\lambda\left(u_{i}\right)} \otimes_{u_{i}+u_{r_{1}}}^{u_{x}} P_{x\left(u_{i}+u_{r_{1}}\right)}^{\lambda} \otimes \cdots \\
& \otimes_{u_{i}+u_{1}+\cdots+u_{p_{p}}}^{u_{i}+u_{p}}+\cdots+P_{x}^{\lambda_{j}\left(u_{i}+u_{r_{1}}+\cdots+u_{p_{p}}\right)}(x(t) \in B)
\end{aligned}
$$

for $i=1, \ldots, n ; x \in X$, where $B$ is any subset of $X$. Here, the notation

$$
\sum_{\left(i, \gamma_{1}, \ldots, \gamma_{\rho} j\right)}^{\prime} \text { for } \rho>1
$$

means that summation is extended over all integers $\gamma_{1}, \ldots, \gamma_{\rho}$ varying from 1 to $n$ such that

$$
i \neq \gamma_{1} \neq \gamma_{2} \neq \cdots \neq \gamma_{\rho-1} \neq j
$$

for $\rho=0$ it means that $i \neq j$, i.e., the sum is empty if $i=j$, and consists of one term if $i \neq j$. The summation

$$
\sum_{\rho=0}^{t-1}
$$

means that $\rho$ varies over $0,1,2, \ldots, t-1$ with one exception: if $i=j$ then there is no term with $\rho=0$ and instead there appears the term

$$
p_{i, i}^{t} P_{x}^{\lambda_{i}}(x(t) \in B)
$$

we refer to this term as the term corresponding to $\rho=-1$. Finally, the notation

$$
\sum_{i}^{\prime}+u_{\gamma_{1}}+\cdots+u_{\gamma_{p}}<t
$$

means that the summation is extended over all integers $u_{i}, u_{\gamma_{1}}, \ldots, u_{\gamma_{\rho}}$ such that

$$
u_{i} \geqslant 1, \quad u_{\gamma_{1}} \geqslant 1, \ldots, u_{\gamma_{\rho}} \geqslant 1, \text { and } u_{i}+u_{\gamma_{1}}+\cdots+u_{\gamma_{\rho}}<t .
$$

The concept of the tensor product

$$
P_{x}^{1} \otimes_{u_{1}}^{0} P_{x\left(u_{1}\right)}^{2} \otimes \cdots \otimes_{u_{1}+\cdots+u_{m}}^{u_{i}+\cdots+u_{m}-1} P_{x\left(u_{1}+\cdots+u_{m}\right)}^{m+1}
$$


used in (1.2) is the same as in [1], [2] (which is taken from [3]) with the obvious adaptation to the discrete parameter case.

Let $\Omega=\Omega_{0} \otimes \Omega_{1}$ and denote by $\mathscr{F}_{t}$ and $\mathfrak{R}_{t}$ the $\sigma$-fields generated by the first $t+1$ coordinates of $\left(x_{0}, x_{1}, x_{2}, \ldots\right)$ and of $\left\{\left(\theta_{0}, \theta_{1}, \theta_{2}, \ldots\right)\right.$, $\left.\left(x_{0}, x_{1}, x_{2}, \ldots\right)\right\}$ respectively.

TheOREM 1.1. The $P_{i, x}$ define a Markov process with respect to $9 \pi_{t}$ and $\Omega$.

Proof. It suffices to verify the Chapman-Kolmogorov equation

$$
\begin{aligned}
& P_{i, x}(\theta(t)=j, x(t) \in B) \\
& \quad=\sum_{l=1}^{n} \sum_{y} P_{i, x}(\theta(s)=l, x(s)=y) P_{l, y}(\theta(t-s)=j, x(t-s) \in B)
\end{aligned}
$$

where $s$ is any integer, $1<s<t-1$, and $B$ is any subset of $X$. The right-hand side of $(1.3)$ is equal to

$$
\begin{aligned}
& \sum_{l=1}^{n} \sum_{y} \sum_{\mu=0}^{s-1} \sum_{\left(i, \alpha_{1}, \ldots, \alpha_{\mu}, l\right)}^{\sum^{\prime}} \sum_{u_{i}+u_{\alpha_{1}}+\cdots+u_{\alpha_{k}}<s} \sum_{\nu=0}^{t-s-1} \sum_{\left(l, \beta_{1}, \ldots, \beta_{\nu} j\right)}^{\prime}
\end{aligned}
$$

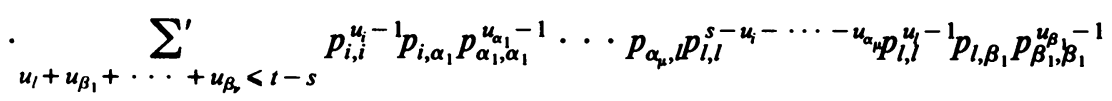

$$
\begin{aligned}
& \cdots p_{\beta_{v}, j} p_{j, j}^{t-s-u_{l}-\cdots-u_{\beta_{v}} P_{x}^{\lambda_{i}} \otimes_{u_{i}}^{0} P_{x}^{\lambda_{q}\left(u_{i}\right)} \otimes \cdots} \\
& \otimes_{u_{i}+\cdots+u_{\alpha_{\mu}}}^{u_{i}+\cdots} P_{x}^{\lambda_{1}\left(u_{i}+\cdots+u_{\alpha_{\mu}}\right)}(x(s)=y) \\
& \cdot P_{y}^{\lambda_{1}} \otimes_{u_{l}}^{0} P_{x}^{\lambda_{(}\left(l_{l}\right)} \otimes \cdots \otimes_{u_{1}+\cdots+u_{\beta_{v}}}^{u_{1}+\cdots} P_{x}^{\lambda_{1}\left(u_{l}+\cdots+u_{\beta_{v}}\right)}(x(t-s) \in B) \text {. }
\end{aligned}
$$

Summing over $y$ and combining the two factors $P^{\lambda_{1}}$ as in [2, following (A.7)] we deduce that the sum over $y$ of the tensor products is equal to

$$
\begin{aligned}
& P_{x}^{\lambda_{i}} \otimes_{u_{i}}^{0} P_{x\left(u_{i}\right)}^{\lambda_{\alpha_{1}}} \otimes \cdots \otimes_{u_{i}+\cdots+u_{\alpha_{\mu}}}^{u_{i}+\cdots+u_{\mu_{j}-1} P_{x\left(u_{i}+\cdots+u_{\alpha_{\mu}}\right.}^{\lambda}} \otimes_{s+u_{i}}^{u_{i}+\cdots+u_{\alpha_{\mu}}} P_{x\left(s+u_{i}\right)}^{\lambda_{\beta_{1}}} \\
& \otimes \cdots \otimes_{s+u_{1}+u_{\beta_{1}}+\cdots+u_{\beta_{p}}+\cdots+u_{\beta_{v}-1} P_{x}^{\lambda_{j}}\left(s+u_{l}+u_{\beta_{1}}+\cdots+u_{\beta_{v}}\right)}(x(t) \in B) .
\end{aligned}
$$

Next we substitute $u_{l}+s \rightarrow u_{l}$. The sum

becomes a sum

$$
\sum_{u_{t}+u_{\beta_{1}}+\cdots+u_{\beta_{2}}<t-s}^{\prime}
$$

$$
\sum_{u_{l}+u_{\beta_{1}}+\cdots+u_{\beta_{2}}<t, u_{l}>s+1}^{\prime}
$$

where the prime " ' " in the last summation indicates that $u_{\beta_{1}} \geqslant 1, \ldots, u_{\beta_{2}} \geqslant$ 1.

We next substitute $u_{l} \rightarrow u_{i}+u_{\alpha_{1}}+\cdots+u_{\alpha_{\mu}}+u_{l}$. The last sum becomes a summation over $u_{l}, u_{\beta_{1}}, \ldots, u_{\beta_{v}}$ subject to

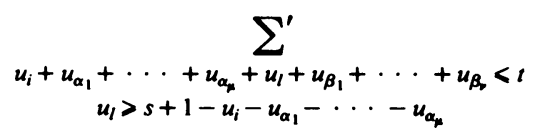


and the prime " "' " indicates that $u_{\beta_{1}} \geqslant 1, \ldots, \mathrm{u}_{\beta_{v}} \geqslant 1$.

The effect of the two substitutions is to transform (1.4) into the sum (cf. [2])

$$
\begin{aligned}
& \sum_{l=1}^{n} \sum_{\mu=0}^{s-1} \sum_{\left(i, \alpha_{1}, \ldots, \alpha_{\mu}, l\right)}^{\prime} u_{u_{i}+u_{\alpha_{1}}+\cdots+u_{\alpha_{k}}<s} \sum_{\nu=0}^{t-s-1} \sum_{\left(l, \beta_{1}, \ldots, \beta_{\nu} j\right)}^{\prime} \\
& \cdot \sum_{u_{i}+u_{\alpha_{1}}+\cdots+u_{\alpha_{i}}+u_{l}+u_{\beta_{1}}+\cdots+u_{\beta_{2}}<t} p_{i, i}^{u_{i}-1} p_{i, \alpha_{1}} \cdots p_{\alpha_{\alpha_{2}}, l} p_{l, l}^{\mu_{l}-1} p_{l, \beta_{1}}^{u_{\beta_{1}}-1} p_{\beta_{1}, \beta_{1}} \cdots \\
& u_{l}>s+1-u_{i}-u_{\alpha_{1}}-\cdots-u_{\alpha_{k}}
\end{aligned}
$$

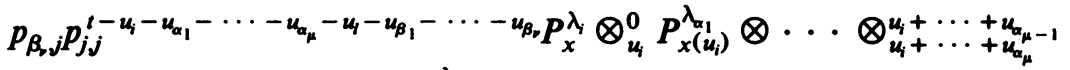

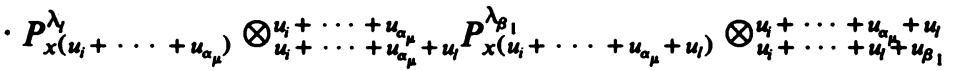

$$
\begin{aligned}
& \cdot P_{x\left(u_{i}+\cdots+u_{l}+u_{\beta_{1}}\right)}^{\lambda_{\beta_{2}}} \otimes \cdots \otimes_{u_{i}+\cdots+u_{\beta_{2}}}^{u_{1}+\cdots} P_{x\left(u_{i}+\cdots+u_{\beta_{2}}\right)}^{\lambda}(x(t) \in B) \text {. }
\end{aligned}
$$

The left-hand side of (1.3) is equal to

$$
\begin{aligned}
& \sum_{\rho=0}^{t-1} \sum_{\left(i, \gamma_{1}, \ldots, \gamma_{\rho} j\right)}^{\prime} \sum_{u_{i}+u_{\gamma_{1}}+\cdots+u_{\gamma_{\rho}}<t} p_{i, i}^{u_{i}-1} p_{i, \gamma_{1}} p_{\gamma_{1}, \gamma_{1}}^{u_{\gamma_{1}}-1} p_{\gamma_{1}, \gamma_{2}} \cdots
\end{aligned}
$$

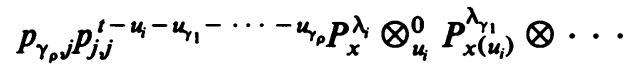

$$
\begin{aligned}
& \otimes_{u_{i}+\cdots+u_{p_{p}}}^{u_{i}+\cdots} P_{x\left(u_{i}+\cdots+u_{p_{p}}\right)}^{\lambda_{j}}(x(t) \in B) .
\end{aligned}
$$

We have to prove that the expressions in (1.5) and (1.6) are equal.

Denote the general term under the summation in (1.6) by

$$
I\left(i, \gamma_{1}, \ldots, \gamma_{\rho}, j ; u_{i}, u_{\gamma_{1}}, \ldots, u_{\gamma_{\rho}}\right) \text {. }
$$

Then the general term under the summation in (1.5) is precisely

$$
I\left(i, \alpha_{1}, \ldots, \alpha_{\mu}, l, \beta_{1}, \ldots, \beta_{v}, j ; u_{i}, u_{\alpha_{1}}, \ldots, u_{\alpha_{\mu}}, u_{l}, u_{\beta_{1}}, \ldots, u_{\beta_{v}}\right) .
$$

Thus it remains to prove the following combinatorial lemma.

LEMMA 1.2. For any positive integers $s, t$ with $s<t$,

$$
\begin{aligned}
& \sum_{l=1}^{n} \sum_{\mu=0}^{s-1} \sum_{\left(i, \alpha_{1}, \ldots, \alpha_{\mu}, l\right)}^{\prime} u_{u_{i}+u_{\alpha_{1}}+\cdots+u_{\alpha_{\mu}}<s} \sum_{\nu=0}^{\prime-s-1} \sum_{\left(l, \beta_{1}, \ldots, \beta_{\nu} j\right)}^{\prime} \\
& \cdot \sum_{\substack{u_{i}+u_{\alpha_{1}}+\cdots+u_{\alpha_{p}}+u_{l}+u_{\beta_{1}}+\cdots+u_{\beta_{2}}<t \\
u_{l}>s+1-u_{i}-u_{\alpha_{1}}-\cdots-u_{\alpha_{p}}}}^{\prime \prime} I\left(i, \alpha_{1}, \ldots, \alpha_{\mu}, l, \beta_{1}, \ldots, \beta_{p}, j ;\right. \\
& \left.u_{i}, u_{\alpha_{1}}, \ldots, u_{\alpha_{x}}, u_{l}, u_{\beta_{1}}, \ldots, u_{\beta_{v}}\right) \\
& =\sum_{\rho=0}^{t-1} \sum_{\left(i, \gamma_{1}, \ldots, \gamma_{\rho} j\right)}^{\prime} \sum_{v_{i}+v_{\gamma_{1}}+\cdots+v_{\gamma_{\rho}}<t}^{\prime} I\left(i, \gamma_{1}, \ldots, \gamma_{\rho}, j ; v_{i}, v_{\gamma_{1}}, \ldots, v_{\gamma_{\rho}}\right) .
\end{aligned}
$$

This lemma is entirely different from the corresponding combinatorial lemma used in [2]. 
Proof of Lemma 1.2. Each term on the left-hand side of (1.7) corresponding to $\mu>0, \nu>0$ appears also on the right-hand side of (1.7) with

$$
\gamma_{k}=\alpha_{k} \quad(1 \leqslant k \leqslant \mu), \quad \gamma_{\mu+1}=l, \quad \gamma_{\mu+m+1}=\beta_{m} \quad(1 \leqslant m \leqslant \nu),
$$

The terms corresponding to $\mu=-1, \nu \geqslant 0$ arise when $l=i$, and then there are no $\alpha$ 's and

$$
u_{i}+u_{\beta_{1}}+\cdots+u_{\beta_{v}} \leqslant t, \quad u_{i} \geqslant s+1 .
$$

These terms also appear on the right-hand side of (1.7) (they are given by (1.8) with no $\alpha$ 's). Similarly, the terms with $\nu=-1, \mu \geqslant 0$ which appear on the left-hand side of (1.7) appear also on the right-hand side. Finally, the term corresponding to $\mu=-1, \nu=-1$ occurs only if $i=j$ and in that case it is precisely the term on the right-hand side of (1.7) corresponding to $\rho=-1$.

It remains to show that each term which appears on the right-hand side of (1.7) with $\rho \geqslant 0$ appears also on the left-hand side and that this correspondence is given by (the one-to-one mapping) (1.8).

Consider the case $\rho>0$. Let

$$
\sigma_{0}=\inf \left\{\sigma ; v_{i}+v_{\gamma_{1}}+\cdots+v_{\gamma_{\sigma}} \geqslant s\right\} .
$$

Suppose first that

$$
v_{i}+v_{\gamma_{1}}+\cdots+v_{\gamma_{o_{0}}}=s .
$$

If $\sigma_{0}<\rho$ then define $\alpha$ 's, $\beta$ 's and $u$ 's by (1.8) with

$$
l=\gamma_{\sigma_{0}+1}, \quad \mu=\sigma_{0}, \quad \nu=\rho-\sigma_{0}-1 .
$$

Since $v_{i}+v_{\gamma_{1}}+\cdots+v_{\gamma_{\mu}}=s$ and $v_{i} \geqslant 1, v_{\gamma_{1}} \geqslant 1, \ldots, v_{\gamma_{\mu}} \geqslant 1$, we have $\mu \leqslant s-1$. Similarly, since

$$
u_{\gamma_{0_{0}+1}}+\cdots+u_{\gamma_{\rho}} \leqslant t-\left(u_{i}+u_{\gamma_{1}}+\cdots+u_{\gamma_{0_{0}}}\right)=t-s
$$

and $\gamma_{m} \geqslant 1$, we must have $\nu \leqslant t-s-1$. Therefore in order for the term $I\left(i, \gamma_{1}, \ldots, \gamma_{\rho}, j ; v_{i}, v_{\gamma_{1}}, \ldots, v_{\gamma_{\rho}}\right)$ to appear on the left-hand side of (1.7) we must show that the restriction

$$
u_{l} \geqslant s+1-u_{i}-u_{\alpha_{1}}-\cdots-u_{\alpha_{\mu}}
$$

is satisfied. But this follows immediately from (1.9) and the fact that $u_{l} \geqslant 1$.

If $\sigma_{0}=\rho$ then the given term appears on the left-hand side of (1.7) with $l=j, \nu=-1$.

So far we have assumed that (1.9) holds. We now assume that (1.9) does not hold, i.e.,

$$
v_{i}+v_{\gamma_{1}}+\cdots+v_{\gamma_{o_{0}}}>s .
$$

If $\sigma_{0}>0$ then we take $l=\gamma_{\sigma_{0}}, \mu=\sigma_{0}-1, \nu=\rho-\sigma_{0}$ in the definition (1.8). Since

$$
u_{i}+u_{\gamma_{1}}+\cdots+u_{\gamma_{o_{0}-1}}<s, \quad u_{i} \geqslant 1, u_{\gamma_{h}} \geqslant 1
$$


we have $\mu \leqslant s-1$. Also

$$
v_{\gamma_{o_{0}+1}}+\cdots+v_{\gamma_{\rho}} \leqslant t-\left(v_{i}+v_{\gamma_{1}}+\cdots+v_{\gamma_{\sigma_{0}}}\right)<t-s
$$

so that $\rho-\sigma_{0}<t-s$, i.e., $\nu \leqslant t-s-1$. Thus it remains to show that

$$
u_{l} \geqslant s+1-u_{i}-u_{\alpha_{1}}-\cdots-u_{\alpha_{i}} \text {. }
$$

But this follows immediately from (1.10).

If $\sigma_{0}=0$ we take $l=i$ and proceed as in the last case. This completes the proof of the lemma.

Having proved Theorem 1.1, we denote by $P^{i, x}$ and $E^{i, x}$ the probabilities and expectations corresponding to the transition probabilities $\boldsymbol{P}_{i, x}$. Recall that the probability space is $\Omega$ and that the $\sigma$-fields are the $: \pi_{t}$.

We shall now extend formula (1.2).

Lemma 1.3. Let $A \in \mathscr{F}_{t}, t=0,1,2, \ldots$ Then

$$
\begin{aligned}
& \int_{A} I_{\theta(t)=j} d P^{i, x}=\sum_{\rho=0}^{t-1} \sum_{\left(i, \gamma_{1}, \ldots, \gamma_{\rho} j\right)}^{\prime} u_{i}+u_{\gamma_{1}}+\cdots+u_{\gamma_{\rho}}<t \\
& p_{i, i}^{u_{1}-1} p_{i, \gamma_{1}} p_{\gamma_{1}, \gamma_{1}}^{u_{\gamma_{1}}-1} p_{\gamma_{1}, \gamma_{2}} p_{\gamma_{2}, \gamma_{2}}^{u_{\gamma_{2}}-1} \ldots p_{\gamma_{\rho} j} p_{j, j}^{t-u_{i}-u_{\gamma_{1}}-\cdots-u_{\gamma_{\rho}} P_{x}^{\lambda_{1}} \otimes P_{x}^{\lambda}\left(u_{j}\right)}
\end{aligned}
$$

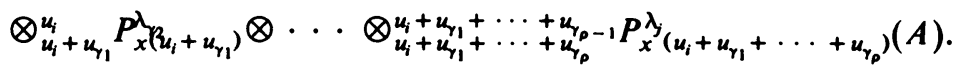

Proof. The proof is similar to the proof of Lemma A.3 in [2]. It suffices to prove (1.11) for a cylindrical set

$$
A=\left(x\left(t_{1}\right) \in B_{1}, \ldots, x\left(t_{m}\right) \in B_{m}\right), \quad t_{1}<t_{2}<\cdots<t_{m} .
$$

Let

$$
\begin{aligned}
A_{i} & =\left(x\left(t_{1}\right) \in B_{1}, \ldots, x\left(t_{i}\right) \in B_{i}\right), \quad 1 \leqslant i \leqslant m, \\
C_{i} & =\left(x\left(t_{i+1}-t_{i}\right) \in B_{i+1}, \ldots, x\left(t_{m}-t_{i}\right) \in B_{m}\right),
\end{aligned}
$$

so that $A=A_{m}$. By the Markov property of the $(x, \theta)$ chain and by (1.2) we obtain, after substituting $u_{l}+t_{m-1} \rightarrow u_{l}$,

$$
\begin{aligned}
& \int_{A} I_{\theta(t)=j} d P^{i, x}=\sum_{l=1}^{n} p_{l, l}^{-t_{m-1}} \int_{A_{m-1}} I_{\theta\left(t_{m-1}\right)=l} \sum_{\nu=0}^{t-1} \sum_{\left(i, \beta_{1}, \ldots, \beta_{\nu}, l\right)}^{\prime} \\
& \cdot \sum_{\substack{u_{l}+u_{\beta_{1}}+\cdots+u_{\beta_{3}<t}<t \\
u_{l}>t_{m-1}+1}} p_{l, l}^{u_{l}-1} p_{l, \beta_{1}} p_{\beta_{1}, \beta_{1}}^{u_{\beta_{1}}-1} \cdots p_{\beta_{r} j} p_{j, j}^{t-u_{l}-\cdots-u_{\beta_{v}} P_{x}^{\lambda_{1}\left(t_{m-1}\right)}}
\end{aligned}
$$

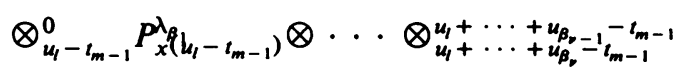

$$
\begin{aligned}
& \text { - } P_{x\left(u_{1}+\cdots+u_{\beta_{v}}-t_{m-1}\right)}^{\lambda_{j}}\left(C_{m}-1\right) d P^{i, x} \text {. }
\end{aligned}
$$


Using the Markov property we can write the right-hand side in the form

$$
\begin{aligned}
& \sum_{k=1}^{n} \int_{A_{m-2}} I_{\theta\left(t_{m-2}\right)=k} \sum_{l=1}^{n} p_{l, l}^{-t_{m-1}} \sum_{\mu=0}^{t_{m-1}-t_{m-2}-1} \sum_{\left(k, \alpha_{1}, \ldots, \alpha_{\mu}, l\right)}^{\prime} \sum_{\nu=0}^{t-t_{m-1} *^{* 1}} \\
& \sum_{\substack{\left(l, \beta_{1}, \ldots, \beta_{p} j\right) \\
u_{k}+u_{\alpha_{1}}+\cdots+u_{\alpha_{k}}<t_{m-1}-t_{m-2}}}^{\sum_{u_{l}+u_{\beta_{1}}+\cdots+u_{\beta_{2}}<t}^{\prime}} \sum_{k, k}^{u_{k}-1} p_{k, \alpha_{1}} p_{\alpha_{1}, \alpha_{1}}^{u_{\alpha_{1}}-1}
\end{aligned}
$$

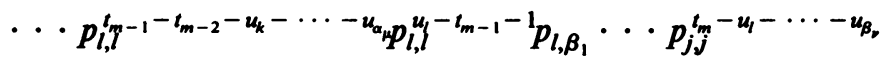

$$
\begin{aligned}
& \cdot P_{x\left(t_{m-2}\right)}^{\lambda_{k}} \otimes_{u_{k}}^{0} P_{x\left(u_{k}\right)_{i}}^{\lambda_{\alpha_{1}}} \otimes \cdots \otimes_{u_{k}+\cdots+u_{\mu_{k}}}^{u_{k}+\cdots+u_{\alpha_{j}-1}} \\
& \cdot P_{x}^{\lambda}\left(u_{k}+\cdots+u_{\alpha_{k}}\right)\left[\left(x\left(t_{m-1}-t_{m-2}\right) \in B_{m-1}\right)\right. \\
& \cdot P_{x\left(t_{m-1}-t_{m-2}\right)}^{\lambda} \otimes_{u_{l}-t_{m-1}}^{0} P_{x\left(u_{l}-t_{m-1}\right)}^{\lambda_{x_{1}}} \otimes \cdots \\
& \left.\otimes_{u_{1}+\cdots+u_{\beta_{v}}+t_{m-1}+t_{m-1}}^{-t_{m-1}} P_{x\left(u_{l}+\cdots+u_{\beta_{v}}-t_{m-1}\right)}^{\lambda_{j}}\left(C_{m-1}\right)\right] d P^{i, x} \text {. }
\end{aligned}
$$

We now make the substitution $u_{k}+t_{m-2} \rightarrow u_{k}$ which transforms

$$
\sum_{u_{k}+u_{\alpha_{1}}+\cdots+u_{\alpha_{k}}<t_{m-1}-t_{m-2}}^{\prime} \text { into } \quad \sum_{\begin{array}{c}
u_{k}+u_{\alpha_{1}}+\cdots+u_{\alpha_{k}}<t_{m-1} \\
u_{k}>t_{m-2}+1
\end{array}}^{\prime}
$$

and then make the substitution $u_{l}-u_{k}-u_{\alpha_{1}}-\cdots-u_{\alpha_{k}} \rightarrow u_{l}$ which transforms

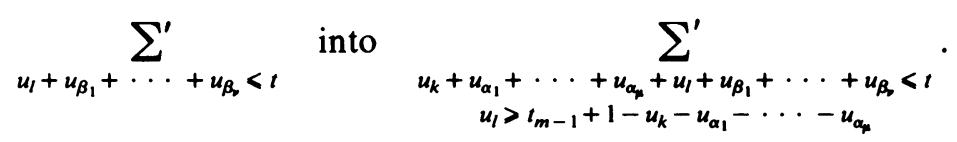

Using the rules (A.16), (A.17) of [2] we finally obtain

$$
\begin{aligned}
& \int_{A} I_{\theta(t)=j} d P^{i, x}=\sum_{k=1}^{n} p_{k, k}^{-t_{m-2}} \int_{A_{m-2}} I_{\theta\left(t_{m-2}\right)=k} \sum_{l=1}^{n} \sum_{\mu=0}^{t_{m-1}-t_{m-2}-1} \\
& \cdot \sum_{\left(k, \alpha_{1}, \ldots, \alpha_{\mu}, l\right)}^{\prime} \sum_{\nu=0}^{t-t_{m-1}-1} \sum_{\left(l, \beta_{1}, \ldots, \beta_{\nu} j\right)} \sum_{\substack{u_{k}+u_{\alpha_{1}}+\cdots+u_{\alpha_{k}}<t_{m-1} \\
u_{k}>t_{m-2}+1}}^{\prime} \\
& \sum_{\substack{u_{k}+u_{\alpha_{1}}+\cdots+u_{\alpha_{k}}+u_{l}+u_{\beta_{1}}+\cdots+u_{\beta_{k}}<t \\
u_{l}>t_{m-1}+1-u_{k}-u_{\alpha_{1}}-\cdots-u_{\alpha_{k}}}}^{\prime} \\
& \cdot\left[p_{k, k}^{u_{k}-1} p_{k, \alpha_{1}} \cdots p_{l, l}^{u_{1}-1} p_{l, \beta_{1}} \cdots p_{j, j}^{l_{j}}-u_{u_{1}}-u_{\alpha_{1}}-\cdots-u_{\beta_{v}}\right. \\
& \cdot P_{x\left(t_{m-2}\right)}^{\lambda_{k}} \otimes_{u_{k}-t_{m-2}}^{0} P_{x\left(u_{k}-t_{m-2}\right)}^{\lambda_{a_{1}}} \otimes \ldots \\
& \otimes_{u_{k}+\cdots+u_{\alpha_{\mu}}=t_{m-2}^{u_{k}}+t_{m-2}}^{u_{t}} P_{x\left(u_{k}+\cdots+u_{\alpha_{k}}-t_{m-2}\right)}^{\lambda_{1}} \otimes \cdots \\
& \left.\otimes_{u_{k}+u_{\alpha_{1}}+\cdots+u_{\beta_{\beta_{v}}}=t_{m-2}}^{u_{k}+u_{\alpha_{1}}+\cdots} P_{x\left(u_{k}+u_{\alpha_{1}}+\cdots+u_{\beta_{v}}-t_{m-2}\right)}^{\lambda_{j}}\left(C_{m-2}\right)\right] d P^{i, x} .
\end{aligned}
$$


We now apply a slightly different version of Lemma 1.2 whereby instead of $t_{m-2}=0$ we have $t_{m-2} \geqslant 0$. We conclude that

$$
\begin{aligned}
\int_{A} I_{\theta(t)=j} d P^{i, x}= & \sum_{k=1}^{n} p_{k, k}^{-t_{m-2}} \int_{A_{m-2}} I_{\theta\left(t_{m-2}\right)=k} \sum_{\rho=0}^{t-t_{m-2}-1} \sum_{\left(k, \gamma_{1}, \ldots, \gamma_{\rho} j\right)}^{\prime} \\
& \cdot \sum_{\substack{u_{k}+\cdots+u_{\gamma_{\rho}}<t \\
u_{k}>t_{m-2}+1}}^{\prime}[\cdots] d P^{i, x}
\end{aligned}
$$

where the expression in [ $\cdots]$ is the same as on the right-hand side of (1.13). Formula (1.14) is analogous to (1.12), except that $m-1$ has been replaced by $m-2$. Proceeding in this way step by step and setting $t_{0}=0, B_{0}=X$, $A_{0}\left(x\left(t_{0}\right) \in B_{0}\right), C_{0}=A$, we arrive at (1.11) with $m-1$ and $t_{m-1}$ replaced by 0 and $t_{0}$ respectively. But this relation is precisely the assertion of the lemma.

2. The $p$-process. In view of the assumption (1.1) we have

$$
\frac{d P_{x}^{\lambda_{j}}}{d P_{x}^{\lambda_{i}}} \mid \mathscr{F}_{t}=\frac{p_{x, x(1)}^{\lambda_{j}} p_{x(1), x(2)}^{\lambda_{1}} \cdots p_{x(t-1), x(t)}^{\lambda_{j}}}{p_{x, x(1)}^{\lambda_{i}} p_{x(1), x(2)}^{\lambda_{i}} \cdots p_{x(t-1), x(t)}^{\lambda_{i}}}
$$

on all paths for which both the numerator and the denominator do not vanish, and $P_{x}^{\lambda_{j}}=0, P_{x}^{\lambda_{1}}=0$ on all the remaining paths. Let

Then we have

$$
z_{i, j}(s, t)=\left.\frac{d P_{x}^{\lambda_{1}} \otimes_{s}^{0} P_{x}^{\lambda_{j}}(s)}{d P_{x}^{\lambda_{i}}}\right|^{\sigma_{t}} \quad(s<t) .
$$

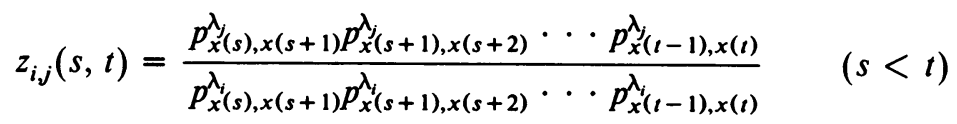

on the paths for which the numerator and denominator do not vanish. Clearly $z_{i, j}(t, t)=1$.

As in [2] we define

We then have (cf. [1], [2])

$$
\bar{p}_{i, j}(t)=P^{i, x}\left[\theta(t)=j \mid \mathscr{F}_{t}\right] \frac{d P^{i, x}}{d P_{x}^{\lambda_{i}}} \mid \widetilde{\mathscr{T}}_{t} .
$$

$$
\begin{aligned}
& \bar{p}_{i, j}(t)=\sum_{\rho=0}^{t-1} \sum_{\left(i, \gamma_{1}, \ldots, \gamma_{\rho} j\right)} \sum_{u_{i}+u_{\gamma_{1}}+\cdots+u_{\gamma_{\rho}}<t}^{\prime} p_{i, i}^{\mu_{i}-1} p_{i, \gamma_{1}} p_{\gamma_{1}, \gamma_{1}}^{u_{\gamma_{1}}-1} p_{\gamma_{1}, \gamma_{2}}
\end{aligned}
$$

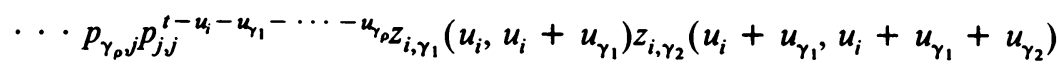

$$
\begin{aligned}
& \cdots z_{i, j}\left(u_{i}+u_{\gamma_{1}}+\cdots+u_{\gamma_{p}}, t\right) \text {. }
\end{aligned}
$$

We now introduce the probabilities

$$
\bar{P}^{p, x}=\sum_{i=1}^{n} p_{i} P^{i, x} \quad\left(p=\left(p_{1}, \ldots, p_{n}\right), p_{i} \geqslant 0, \sum_{i=1}^{n} p_{i}=1\right)
$$


and the process

$$
X(t)=\left(x_{x}(t), p_{1}(p, t), \ldots, p_{n}(p, t)\right)
$$

where $t=0,1,2, \ldots ; x_{x}(t)$ is $x(t)$ with $x(0)=x$ and

$$
p_{j}(p, t)=\bar{E}^{p, x}\left[\theta(t)=j \mid \mathscr{F}_{t}\right] .
$$

Here $\bar{E}^{p, x}$ is the expectation corresponding to the probability $\bar{P}^{p, x}$. As in [2] we have

$$
p_{j}(p, t)=\sum_{i=1}^{n} p_{i} \bar{p}_{i, j}(t) /\left[\sum_{l=1}^{n} p_{l} z_{i, l}(0, t) \sum_{k=1}^{n} \bar{p}_{l, k}(t)\right] .
$$

THEOREM 2.1. The process $X(t)$ is a Markov process, with respect to the $\sigma$-fields $\mathscr{F}_{t}$ and the measures $\bar{P}^{p, x}$.

The proof is similar to the proof of the corresponding result in the Appendix of [2] except that now we use Lemma 1.3 instead of Lemma A.2 of [2].

3. The quality control problem. Using the notation $(0.1)$, we introduce the cost function (0.2) and, more generally, the cost

$$
\begin{aligned}
J_{x}^{p}(\tau)= & \bar{E}^{p, x}\left[K(p)+\sum_{l=1}^{\infty} K\left(\theta\left(\tau_{l}\right)\right) I_{\theta\left(\tau_{l}\right) \neq n}\right] \\
& +\bar{E}^{p, x}\left[\int_{0}^{\tau_{1}-1} f(\theta(s)) d s+\sum_{l=1}^{\infty} I_{\theta\left(\tau_{l}\right) \neq n} \int_{\tau_{l}-1}^{\tau_{l+1}-1} f(\theta(s)) d s\right]
\end{aligned}
$$

where $K(p)=K_{i}$ if $p=\left(p_{1}, \ldots, p_{n}\right), p_{1}=\cdots=p_{i-1}=0, p_{i} \neq 0$; if the process $\theta(t)$ is such that $p_{i, j}(t)=0$ whenever $j<i$ then no restrictions are made on the $K_{i}$, but if the process $\theta(t)$ can go in both directions then we require that $K_{1}=K_{2}=\cdots=K_{n-1}$. Here $\tau=\left(\tau_{1}, \tau_{2}, \ldots\right)$ is a sequence of inspection times, i.e.,

$$
\tau_{1}=\sigma_{1}, \quad \tau_{m+1}=\tau_{m}+\sum_{l=1}^{n-1} I_{\theta\left(\tau_{m}\right)=l} \sigma_{m, l}\left(\phi_{\tau_{m}}\right) \quad(m \geqslant 1)
$$

where $\sigma_{1}, \sigma_{m, l}$ are stopping times with respect to $\widetilde{F}_{t}$ with nonnegative integer values, and $\phi$ is the shift operator: $\phi_{s} x(t)=x(t+s)$. It is understood that $\tau_{m+i}=\infty(i>1)$ on the set $\tau_{m}=\infty$. Also, in (3.1), $K\left(\theta\left(\tau_{l}\right)\right) I_{\theta\left(\tau_{l}\right) \neq n}$ and $\int_{\tau_{l}-1}^{\tau_{\tau_{1}}-1} f(\theta(s)) d s$ do not appear whenever $\tau_{l}=\infty$. We shall denote by $\mathbb{Q}$ the class of all sequences of inspection times. We are interested in the problem of characterizing $\bar{\tau}_{p} \in \mathbb{Q}$ such that

$$
J_{x}^{p}\left(\bar{\tau}_{p}\right)=\inf _{\tau \in \mathbb{Q}^{\mathcal{P}}} J_{x}^{p}(\tau) .
$$

Denote by $A_{x, p}$ the generator of the Markov process occurring in Theorem 
2.1. Thus, $A_{x, p}$ is defined by

$$
A_{x, p} u(x, p)=\bar{E}^{p, x}[u(x(1), p(p, 1))-u(x, p)]
$$

where $p(p, t)=\left(p_{1}(p, t), \ldots, p_{n}(p, t)\right)$.

Using the Markov property one can establish, by induction on $t(t=1$, $2, \ldots$ ), Dynkin's formula

$$
\begin{aligned}
\bar{E}^{p, x}\left[u\left(x_{x}(t), p(p, t)\right)\right]-u(x, p) \\
\quad=\bar{E}^{p, x}\left[\int_{0}^{t-1} A_{x_{x}(s), p(p, s)} u\left(x_{x}(s), p(p, s)\right) d s\right] .
\end{aligned}
$$

We can now proceed as in [2] to reduce the problem of characterizing an optimal $\bar{\tau}_{p}$ as in (3.3) to the problem of solving the following quasi variational inequality (q.v.i.) for a function $V(x, p)$ :

$$
V(x, p) \leqslant K(p)+\sum_{j=1}^{n-1} p_{j} V\left(x, e_{j}\right)
$$

where $e_{j}$ is the $j$ th unit vector $(0,0, \ldots, 0,1,0, \ldots, 0)$,

$$
\begin{gathered}
A_{x, p} V(x, p)+\sum_{j=1}^{n} c_{j} p_{j}>0 \\
{\left[A_{x, p} V(x, p)+\sum_{j=1}^{n} c_{j} p_{j}\right]\left[K(p)+\sum_{j=1}^{n-1} p_{j} V\left(x, e_{j}\right)-V(x, p)\right]=0}
\end{gathered}
$$

where the $p_{j}$ vary in the set $p_{j} \geqslant 0, \sum_{j=1}^{n} p_{j}=1$ and $x$ varies in $X$.

Let

$$
\begin{aligned}
S=\left\{(x, p) ; x \in X, p=\left(p_{1}, \ldots, p_{n}\right), p_{j}\right. & \geqslant 0, \sum_{j=1}^{n} p_{j}=1, \\
V(x, p) & \left.=K(p)+\sum_{j=1}^{n-1} p_{j} V\left(x, e_{j}\right)\right\} .
\end{aligned}
$$

Define the $\mathscr{F}_{t}$ stopping times:

$$
\begin{aligned}
& \sigma_{*}^{p}=\text { hitting time of the set } S \text { by } X(t)=(x(t), p(p, t)), \\
& \sigma_{*}^{l}=\sigma_{*}^{p} \text { when } p=e_{l}, \\
& \bar{\tau}_{1}^{p}=\sigma_{*}^{p}, \bar{\tau}_{m+1}^{p}=\bar{\tau}_{m}^{p}+\sum_{l=1}^{n-1} I_{\theta\left(\bar{\tau}_{m}^{p}\right)=l} \sigma_{*}^{l}\left(\phi_{\bar{\tau}_{m}^{p}}^{p}\right), \\
& \bar{\tau}^{p}=\left(\bar{\tau}_{1}^{p}, \bar{\tau}_{2}^{p}, \bar{\tau}_{3}^{p}, \ldots\right) .
\end{aligned}
$$

THEOREM 3.1. Let $V(x, p)$ be a solution of the q.v.i. (3.6) - (3.8). If the $\sigma_{*}^{p}$ 
are finite valued then

$$
V(x, p)=\inf _{\tau \in Q} J_{x}^{p}(\tau)=J_{x}^{p}\left(\bar{\tau}^{p}\right) .
$$

The proof is similar to the proof of the corresponding result in [2] and will therefore be omitted.

In the special case where

$$
p_{i, j}=0 \text { if } 1 \leqslant j<i \leqslant n
$$

the q.v.i. reduces to a sequence of simpler q.v.i. analogous to (4.35)-(4.37) in [2].

Another type of simplification of (3.6)-(3.8) occurs when

$$
p_{x(s), x(t)}^{\lambda_{i}}=p_{x(0), x(t-s)}^{\lambda_{i}} \equiv p_{t-s}^{\lambda_{1}} \quad \text { if } 0 \leqslant s<t .
$$

In this case the numbers

$$
\bar{P}^{p, x}\left[p_{j}(p, t) \in B ; 1 \leqslant j \leqslant n\right]
$$

do not depend on $x$ and, consequently, the process

$$
p_{j}(p, t) \quad(1 \leqslant j \leqslant n) \text { with measures } \bar{P}^{p, 0}
$$

is a Markov process. We shall denote its generator by $A_{p}$.

Denote by $R_{i}^{*}$ the (countable) range of the process $\left(p_{j}\left(e_{i}, t\right) ; 1 \leqslant j \leqslant n\right)$ and let $R^{*}=\cup_{i=1}^{n-1} R_{i}^{*}$.

One is interested in the quality control problem mainly for the initial values $p=e_{i}$. In case (3.13) holds it then suffices to solve the q.v.i. in the set $R^{*}$ only. Thus we have to solve a "discrete" q.v.i.

In the next section we shall solve the discrete q.v.i. in a case when $n=2$.

4. Solution of the discrete q.v.i. in case $n=2$. We assume that (3.12), (3.13) hold and that $n=2$. Thus $p_{2,1}=0, p_{2,2}=1$. To rule out a trivial case, we assume that $p_{1,1}>0, \mathrm{p}_{1,2}>0$.

Let

$$
p_{j}^{\lambda_{1}}=p_{i, i+j}^{\lambda_{1}}, \quad p_{j}^{\lambda_{2}}=p_{i, i+j}^{\lambda_{2}}
$$

where $p_{k, l}^{\lambda_{k}}$ is the transition probability matrix of $P_{x}^{\lambda_{k}}$. Denote by $N$ the set of $j$ 's for which $p_{j}^{\lambda_{i}} \neq 0$, and let

Since

$$
\mu_{j}=p_{j}^{\lambda_{2}} / p_{j}^{\lambda_{1}} \quad(j \in N) .
$$

we must have

$$
\sum_{j \in N} p_{j}^{\lambda}=1 \quad(i=1,2),
$$

$$
\sum_{j \in N} p_{j}^{\lambda_{1}} \mu_{j}=1
$$

Since $p_{2,1}=0, p_{2,2}=1$, we have

$$
\bar{p}_{1,1}(1)=p_{1,1}, \quad \bar{p}_{1,2}(1)=p_{1,2}, \quad \bar{p}_{2,1}(t)=0, \bar{p}_{2,2}(t)=1,
$$


Hence, (2.9) for $t=1$ simplifies to

$$
p_{1}(p, 1)=\frac{p_{1} p_{1,1}}{p_{1}+p_{2} \mu_{x(1)-x(0)}}, \quad p_{2}(p, 1)=\frac{p_{1} p_{1,2}+p_{2} \mu_{x(1)-x(0)}}{p_{1}+p_{2} \mu_{x(1)-x(0)}} .
$$

Defining

$$
T_{j}^{1}\left(p_{1}, p_{2}\right)=\frac{p_{1} p_{1,1}}{p_{1}+p_{2} \mu_{j}}, \quad T_{j}^{2}\left(p_{1}, p_{2}\right)=\frac{p_{1} p_{1,2}+p_{2} \mu_{j}}{p_{1}+p_{2} \mu_{j}},
$$

we can write the generator $A_{p}\left(p=p_{1}, p_{2}\right)$ in the form

$$
\begin{aligned}
A_{p} g(p) & =\bar{E}^{p}[g(p(p, 1))-g(p)] \\
& =p_{1} \bar{E}^{e_{1}}[g(p(p, 1))-g(p)]+p_{2} \bar{E}^{e_{2}}[g(p(p, 1))-g(p)] \\
& =\bar{E}^{e_{1}}\left[\left(p_{1}+p_{2} z_{1,2}(0,1)\right)(g(p(p, 1))-g(p))\right] \\
& =\sum_{j \in N} p_{j}^{\lambda_{1}}\left(p_{1}+p_{2} \mu_{j}\right)\left[g\left(T_{j}^{1}\left(p_{1}, p_{2}\right), T_{j}^{2}\left(p_{1}, p_{2}\right)\right)-g\left(p_{1}, p_{2}\right)\right]
\end{aligned}
$$

where we have used the facts

$$
\bar{E}^{e_{1}}[g]=\bar{E}^{e_{2}}\left[g z_{1,2}(0,1)\right] \quad(g=g(p(p, 1))), \quad z_{1,2}(0,1)=\mu_{x(1)-x(0)}
$$

and the notation $e_{1}=(0,1), e_{2}=(1,0)$.

Define

$$
y=p_{1} / p_{2}, \quad g(y)=g\left(p_{1}, p_{2}\right) .
$$

Then, as easily verified,

$$
A_{p} g\left(p_{1}, p_{2}\right)=L_{y} g(y)
$$

where

$$
L_{y} g(y)=\frac{1}{1+y} \sum_{j \in N} p_{j}^{\lambda_{1}}\left(1+y \mu_{j}\right)\left[g\left(T_{j}(y)\right)-g(y)\right]
$$

and

$$
T_{j}(y)=\frac{p_{1,2}}{p_{1,1}}+\frac{\mu_{j}}{p_{1,1}} y \quad(j \in N) .
$$

We shall impose the monotonicity condition

$$
T_{j}(y)>y \quad(j \in N) \text {. }
$$

that is

$$
\mu_{j} \geqslant p_{1,1} \quad(j \in N) .
$$

This condition implies that

$$
\frac{p_{1}(p, t)}{p_{2}(p, t)}>\frac{p_{1}(p, s)}{p_{2}(p, s)} \quad \text { if } t>s .
$$


It is easily verified that

$$
A_{p} p_{2}=-p_{1,2} p_{2}
$$

We shall need Dynkin's formula

$$
\bar{E}^{e_{1}}[g(p(\tau))]-g(p)=\bar{E}^{e_{1}}\left[\int_{0}^{\tau-1} A_{p} g(p(s)) d s\right] \quad\left(p(0)=e_{1}\right)
$$

where $g$ is any function defined on the discrete set $R^{*}$ and $\tau$ is any bounded $\pi$, stopping time.

Let $b$ be any positive number and let $\tau_{b}$ be the first time such that $\left(p_{1}(t) / p_{2}(t)\right) \geqslant b$. (Notice $p_{1}\left(\tau_{b}\right) / p_{2}\left(\tau_{b}\right)$ is not necessarily equal to $b$.) Applying (4.12) with $g\left(p_{1}, p_{2}\right)=p_{2}$ and $\tau=\tau_{b} \wedge m(m>0)$ and using (4.11), we conclude that

$$
\bar{E}^{e_{1}}\left[\tau_{b} \wedge m\right] \leqslant C
$$

where $C$ is a constant independent of $M$. Taking $m \rightarrow \infty$ we conclude that

$$
\bar{E}^{e_{1}}\left[\tau_{b}\right]<\infty \text {. }
$$

Notice that the proof of (4.13) does not exploit the monotonicity assumption (4.9).

We now assume for simplicity that $c_{1}=0$ (but $c_{2}>0$ ), and set $c=c_{2}$, $K=K_{1}$.

Recalling (4.6), (4.7), the q.v.i. (3.6)-(3.8) can be written in terms of the function $V(y)=V\left(p_{1}, p_{2}\right)\left(y=p_{1} / p_{2}\right)$ in the form

$$
\begin{gathered}
L_{y} V(y)+\frac{c y}{1+y} \geqslant 0 \quad \text { in } \hat{R}, \\
V(y) \leqslant K+\frac{V(0)}{1+y} \text { in } \hat{R}, \\
{\left[L_{y} V(y)+\frac{c y}{1+y}\right]\left[K+\frac{V(0)}{1+y}-V(y)\right]=0 \text { in } \hat{R}}
\end{gathered}
$$

where $\hat{R}$ is the (discrete) range of $p_{1}(t) / p_{2}(t)$ when $p_{1}(0)=0, p_{2}(0)=1$.

Theorem 4.1. Let (4.1), (4.9) hold. Then there exist a unique $b \in \hat{R}, b>0$ and a unique function $V(y)$ defined on $\hat{R}$ such that

$$
\begin{gathered}
L_{y} V(y)+\frac{c y}{1+y}=0 \quad \text { if } y \in \hat{R}, 0 \leqslant y<b, \\
L_{y} V(y)+\frac{c y}{1+y}>0 \quad \text { if } y \in \hat{R}, y>b, \\
V(y)=K+\frac{V(0)}{1+y} \quad \text { if } y \in \hat{R}, y \geqslant b, \\
V(y)<K+\frac{V(0)}{1+y} \quad \text { if } y \in \hat{R}, 0 \leqslant y<b .
\end{gathered}
$$


Notice that $V(y)$ is then a solution of the q.v.i. (4.14)-(4.16). In view of (4.13), $\tau_{b}<\infty$ and therefore Theorem 3.1 can be applied to conclude that

$$
V(0)=\inf _{\tau \in \mathscr{Q}} J_{x}^{1}(\tau)=J_{x}^{1}\left(\bar{\tau}^{1}\right) .
$$

The optimal inspection is then to inspect at time $\tau_{b}$ (given that $p(0)=(0,1)$ ); let $p(t)$ start again at $p(0)=(0,1)$ and again inspect at time $\tau_{b}$, etc.

Proof of Theorem 4.1. Suppose $b$ is such that (4.17), (4.19) hold. By Dynkin's formula we then get (with $E=\bar{E}^{e_{1}}, P=\bar{P}^{e_{1}}, y(t)=p_{1}(t) / p_{2}(t)$ )

$$
E\left[K+\frac{V(0)}{1+y\left(\tau_{b}\right)}\right]-V(0)=E\left[-\int_{0}^{\tau_{b}-1} \frac{c y(s)}{1+y(s)} d s\right],
$$

or

$$
V(0) E\left[\frac{y\left(\tau_{b}\right)}{1+y\left(\tau_{b}\right)}\right]=K+c E\left[\int_{0}^{\tau_{b}-1} \frac{y(s)}{1+y(s)} d s\right]
$$

Setting

$$
\begin{gathered}
H(b, d z)=P\left[y\left(\tau_{b}\right) \in z\right], \\
L(b, d z)=\sum_{s<\tau_{b}-1} P[y(s) \in z],
\end{gathered}
$$

we then obtain from (4.22) an expression for $V(0)$ :

$$
V(0)=\frac{K+c \int \frac{z}{1+z} L(b, d z)}{\int \frac{z}{1+z} H(b, d z)} \equiv Q(b) .
$$

Notation. For any $b \in \hat{R}$ we denote by $\hat{b}$ the number in $\hat{R}$ immediately to the right of $b$.

Lemma 4.2. For any $b \in \hat{R}$,

$$
L(\hat{b}, d b)=P\left(y\left(\tau_{b}\right)=b\right) .
$$

Proof. The left-hand side is equal to

$$
\begin{aligned}
\sum_{s<\tau_{b}-1} P(y(s)=b)= & \sum_{s<\tau_{b}-1} P(y(s)=b) \\
& +\sum_{\tau_{b}-1<s<\tau_{\dot{b}}-1} P(y(s)=b) .
\end{aligned}
$$

The first sum on the right-hand side is equal to zero (by the definition of $\tau_{b}$ ). Since $\tau_{\hat{b}}-\tau_{b} \leqslant 1$ by the monotonicity assumption, and $\tau_{\hat{b}}-\tau_{b}=1$ if and only if $y\left(\tau_{b}\right)=b$, the second sum on the right-hand side of (4.27) is equal to

$$
\sum_{s=\tau_{b}} P(y(s)=b)=P\left(y\left(\tau_{b}\right)=b\right),
$$

and (4.26) follows. 
We also have the relation

$$
L(\hat{b}, d b)=H(b, d b)
$$

however this relation will not be needed.

LEMMA 4.3. The following formula holds.

$$
\int \frac{z}{1+z} H(b, d z)=p_{1,2} \int \frac{1}{1+z} L(b, d z) .
$$

Proof. Since $L_{y}(1)=0$,

$$
L_{y}\left(\frac{y}{1+y}\right)=-L_{y}\left(\frac{1}{1+y}\right)=\frac{p_{1,2}}{1+y}
$$

by (4.11). Hence, by Dynkin's formula,

$$
E\left[\frac{y\left(\tau_{b}\right)}{1+y\left(\tau_{b}\right)}\right]=E\left[\int_{0}^{\tau_{b}-1} \frac{p_{1,2}}{1+y(s)} d s\right] .
$$

Recalling (4.23) and (4.24), (4.29) follows.

Using Lemma 4.3, we can rewrite the expression $Q(b)$ introduced in (4.25) in the form

$$
Q(b)=\frac{K+c \int \frac{z}{1+z} L(b, d z)}{p_{1,2} \int \frac{1}{1+z} L(b, d z)} .
$$

Our plan now is to show that there is a unique $b$ which minimizes $Q(b)$ and then show that the function $V$ defined by (4.17), (4.19) also satisfies (4.18), (4.20). The uniqueness of the minimal $b$ implies the uniqueness assertion of Theorem 4.1 .

For any $b \in \hat{R}$, we compute from (4.30)

$$
\begin{gathered}
Q(\hat{b})-Q(b)=\left[c \int \frac{z}{1+z} L(\hat{b}, d z)-c \int \frac{z}{1+z} L(b, d z)\right] \\
/\left[p_{1,2} \int \frac{1}{1+z} L(\hat{b}, d z)\right] \\
+\left[K+c \int \frac{z}{1+z} L(b, d z)\right] \frac{1}{p_{1,2}}\left[\int \frac{1}{1+z} L(b, d z)\right. \\
\left.-\int \frac{1}{1+z} L(\hat{b}, d z)\right] \\
/\left[\left(\int \frac{1}{1+z} L(\hat{b}, d z)\right) \cdot\left(\int \frac{1}{1+z} L(b, d z)\right)\right] .
\end{gathered}
$$

By the strict monotonicity of the $y$-process

$$
\int_{z<y} h(z) L(\overline{\bar{y}}, d z)=\int_{z<y} h(z) L(\bar{y}, d z) \quad \text { if } y<\bar{y}<\overline{\bar{y}} .
$$


Hence

$$
\int \frac{1}{1+z} L(\hat{b}, d z)-\int \frac{1}{1+z} L(b, d z)=\frac{1}{1+b} L(\hat{b}, d b) .
$$

Since $b \in \hat{R}$ and the $y$-process is monotone, $P\left(y\left(\tau_{b}\right)=b\right)>0$ so that, by Lemma 4.2, also $L(\hat{b}, d b)>0$. It follows that

$$
\begin{aligned}
& \operatorname{sgn}[Q(\hat{b})-Q(b)] \\
& \quad=\operatorname{sgn}\left[c b \int \frac{1}{1+z} L(b, d z)-c \int \frac{z}{1+z} L(b, d z)-K\right] \\
& \quad=\operatorname{sgn}\left[c \int \frac{b-z}{1+z} L(b, d z)-K\right]
\end{aligned}
$$

Let

$$
S(b)=\int \frac{b-z}{1+z} L(b, d z)
$$

Then, using (4.32),

$$
\begin{aligned}
S(\hat{b})-S(b) & =\int \frac{\hat{b}-z}{1+z} L(\hat{b}, d z)-\int \frac{b-z}{1+z} L(b, d z) \\
& =\int \frac{\hat{b}-b}{1+z} L(b, d z)+\frac{1}{1+b} L(\hat{b}, d b) .
\end{aligned}
$$

The right-hand side is larger than $(\hat{b}-b) L(b, d 0)=\hat{b}-b$. Hence $S(b)$ is strictly increasing on $\hat{R}$ and $S(b) \rightarrow \infty$ if $b \rightarrow \infty$.

From (4.33), (4.34) we then conclude that there is a unique $b$ in $\hat{R}$ such that

$$
\begin{array}{ll}
Q(\hat{y})-Q(y)<0 & \text { if } y<b, y \in \hat{R}, \\
Q(\hat{y})-Q(y)>0 & \text { if } y \geqslant b, y \in \hat{R} .
\end{array}
$$

The point $b$ is then the unique minimum of $Q(y), y \in \hat{R}$.

We next define $V(y)$ for $y \geqslant b, y \in \hat{R}$ by (4.19) and for $0<y<b, y \in \hat{R}$ by (4.17) (using iteration and the strict monotonicity of the $y$-process). It remains to show that (4.18) and (4.20) hold.

To prove (4.18) we compute

$$
L_{y}\left(K+\frac{V(0)}{1+y}\right)=\frac{c y}{1+y}-\frac{V(0) p_{1,2}}{1+y} .
$$

Thus, (4.18) would follow from

$$
c b-V(0) p_{1,2}>0 \text {. }
$$

If we prove that

$$
L_{y}\left(V(y)-K-\frac{V(0)}{1+y}\right)<0 \text { if } y \in \hat{R}, y<b
$$


then by the maximum principle (which holds, since the $y$-process is monotone) and the fact that

$$
V(y)-K-\frac{V(0)}{1+y} \begin{cases}=0 & \text { if } y=b \\ <0 & \text { if } y=0\end{cases}
$$

it follows that (4.20) holds. Since the left-hand side of (4.38) is equal to $c y-V(0) p_{1,2},(4.20)$ is thus a consequence of

$$
c y-p_{1,2} V(0)<0 \text { if } y \in \hat{R}, y<b .
$$

Thus, to complete the proof of the theorem it remains to prove (4.37), (4.39). Inserting $V(0)$ from (4.25) (or rather (4.30)) into (4.37), (4.39) we find that these two inequalities reduce to

$$
\begin{aligned}
& c b \int \frac{1}{1+z} L(b, d z)-\left(K+c \int \frac{z}{1+z} L(b, d z)\right)>0, \\
& c y \int \frac{1}{1+z} L(b, d z)-\left(K+c \int \frac{z}{1+z} L(b, d z)\right)<0 \quad(y<b) ;
\end{aligned}
$$

but these inequalities clearly follow from (4.33) and (4.36).

\section{REFERENCES}

1. R. F. Anderson and A. Friedman, A quality control problem and quasi variational inequalities, Arch. Rational Mech. Anal. 63 (1977), 205-252.

2. Multi-dimensional quality control problems and quasi variational inequalities, Trans. Amer. Math. Soc. 246 (1978), 31-76.

3. D. W. Stroock and S. R. S. Varadhan, Diffusion processes with continuous coefficients. I, Comm. Pure Appl. Math. 22 (1969), 345-400. MR 40 \#6641.

Department of Mathematics, University of Pittsburgh, Pittsburgh, Pennsylvania 15260

Department of Mathematics, Northwestern University, Evanston, Illinois 60201 